\title{
Inducible metabolic pathway for citrate metabolism in case of major liver dysfunction: fact or fiction?
}

\author{
Patrick M. Honore ${ }^{1 *}$, David De Bels ${ }^{1}$, Sebastien Redant ${ }^{1}$, Rachid Attou' ${ }^{1}$, Luc Kugener ${ }^{1}$ and Willem Boer ${ }^{2}$ \\ This comment refers to the article available at https://doi.org/10.1186/s13054-017-1870-3.
}

With interest, we read the paper published by Klingele et al. [1] in a recent issue of critical care.

Because citrate metabolism is oxygen dependent via the citric acid cycle and therefore mainly takes place in organs with high amounts of mitochondria, such as the liver, kidney, or muscle [1], they hypothesized that adequate citrate metabolism must be possible outside the liver, implying an inducible metabolic pathway. They postulate that disturbed microvascular circulation could cause altered hepatic function, resulting in the association of impaired liver function and citrate accumulation.

In neonates with mitochondrial cytopathy [2], the citric acid cycle does not function properly and therefore anticoagulation with citrate is contraindicated. We report two cases of neonates suffering from mitochondrial cytopathy in whom unfractionated heparin was stopped because of bleeding complications. Circuit half-lives without anticoagulation were short $(8 \mathrm{~h})$. To remedy this, it was decided to introduce citrate slowly but progressively, increasing the dose, monitoring for accumulation every $2 \mathrm{~h}$. After $96 \mathrm{~h}$, the two neonates were able to metabolize citrate despite citric acid cycle dysfunction, inferring activation of the inducible pathway.

It is well known that in all cells, citrate can be metabolized within the Cori cycle (tricarboxylic acid cycle) [1]. It describes the linked metabolic pathways by which muscles, even in the absence of oxygen, remain capable of functioning, producing lactate. It is activated both by citrate [2] and adrenaline, the latter leading to the production of lactate [3]. The muscles normally combine glucose with oxygen to generate energy. In anaerobic circumstances, lactate is produced which is synthesized by the liver through gluconeogenesis, the process of glucose production from non-carbohydrate components.

In the setting of citrate anticoagulation, with disturbed microvascular circulation causing altered hepatic function and citrate accumulation [1], it is also our experience that lactate better reflects citrate accumulation than calcium ratio and liver dysfunction itself. However, in a recent review, Schneider et al. concluded the opposite [4]. For them, lactate elevation is not secondary to citrate accumulation but due to a common primary process impairing the tricarboxylic acid cycle, reducing citrate metabolism, and limiting pyruvate metabolism leading to lactate generation [4]. The liver citrate anticoagulation threshold (L-CAT) trial showed the safety of continuous renal replacement therapy (CRRT)- citrate in patients with severely impaired liver function [5]. We conclude that Cori cycle is functional without oxygen [2] and that citrate metabolism is less dependent on the hepatic function itself and more dependent upon the whole microcirculation of the body as suggested by Klingele [1].

\footnotetext{
* Correspondence: Patrick.Honore@CHU-Brugmann.be

${ }^{1}$ ICU Department, Centre Hospitalier Universitaire Brugmann, 4,Place Van

Gehuchten, 1020 Brussels, Belgium

Full list of author information is available at the end of the article
} 


\section{Authors' response}

\section{Matthias Klingele, Theresa Stadler, Danilo Fliser, Timo Speer, Heinrich V. Groesdonk and Alexander Raddatz}

Honore and colleagues report about two neonates suffering from mitochondrial cytopathy being able to metabolize citrate despite citric acid cycle dysfunction. They conclude from this interesting observation that this infers activation of an inducible pathway, and they refer to our study [1].

We reported about long-term continuous renal replacement therapy (RRT) and anticoagulation with citrate (RCA) in critically ill patients with severe liver dysfunction and unexpected low rates of citrate accumulation. Thus, the citrate metabolism in extrahepatic organs with high amounts of mitochondria such as the muscles was mostly sufficient to deal with the amount of citrate delivered by CRRT with RCA. We therefore concluded that a substantial extrahepatic metabolism of citrate seems to exist. However, in case of disturbed microcirculation, both hepatic and extrahepatic citrate metabolisms were clearly disturbed [1]. In a systematic review, Zangh et al. confirm our findings. They could demonstrate that patients with and without liver dysfunction showed similar laboratory parameters during CRRT with RCA, indicating a comparable citrate metabolism in patients with and without liver dysfunction [6].

Our hypothesis was that the risk of citrate accumulation during regional citrate anticoagulation is more dependent on microcirculation than on liver function. Disturbed microcirculation results in elevated lactate due to impaired organic substrate metabolism. Therefore, lactate is a risk factor for impaired citrate metabolism. This is supported by Khadzhynov and colleagues [7]. However, they suggest that lactate kinetics rather than initially elevated lactate concentration should be considered in assessing the risk of citrate accumulation.

Honore and colleagues agree that disturbed microvascular circulation and lactate are a hint for citrate accumulation and that extrahepatic citrate metabolism explains why CRRT with RCA in case of liver dysfunction not necessarily results in citrate accumulation. Moreover, they suggest an inducible pathway which can be activated despite mitochondrial cytopathy and citric acid cycle dysfunction. This is an interesting and novel approach, being able to explain why hepatic function may be less important for citrate metabolism in CRRT with RCA than previously thought.

\section{Abbreviations}

CRRT: Continuous renal replacement therapy; L-CAT: Liver citrate anticoagulation threshold
Funding

None.

Availability of data and materials

Not applicable.

Authors' contributions

PMH and WB designed the paper. All authors participated in drafting and reviewing. All authors read and approved the final version of the manuscript.

Ethics approval and consent to participate

Not applicable.

Consent for publication

Not applicable.

\section{Competing interests}

The authors declare to have no competing interests.

\section{Publisher's Note}

Springer Nature remains neutral with regard to jurisdictional claims in published maps and institutional affiliations.

\section{Author details}

${ }^{1}$ ICU Department, Centre Hospitalier Universitaire Brugmann, 4,Place Van Gehuchten, 1020 Brussels, Belgium. ²Department of Anesthesiology, Intensive Care Medicine, Emergency Medicine \& Pain Medicine, Ziekenhuis

Oost-Limburg, Genk, Belgium.

Received: 14 February 2019 Accepted: 10 April 2019

Published online: 14 May 2019

\section{References}

1. Klingele M, Stadler T, Fliser D, Speer T, Groesdonk HV, Raddatz A. Long-term continuous renal replacement therapy and anticoagulation with citrate in critically ill patients with severe liver dysfunction. Crit Care. 2017;21:294.

2. Holmes RD, Moore KH, Ofenstein JP, Tsatsos P, Kiechle FL. Lactic acidosis and mitochondrial dysfunction in two children with peroxisomal disorders. $J$ Inherit Metab Dis. 1993;16(2):368-80.

3. Kusaka M, Ui M. Activation of the Cori cycle by epinephrine. Am J Phys. 1977;232:E145-55.

4. Schneider AG, Journois $D$, Rimmelé T. Complications of regional citrate anticoagulation: accumulation or overload? Crit Care. 2017;21:281

5. Slowinski T, Morgera S, Joannidis M, Henneberg T, Stocker R, Helset E, et al. Safety and efficacy of regional citrate anticoagulation in continuous venovenous hemodialysis in the presence of liver failure: the liver citrate anticoagulation threshold (L-CAT) observational study. Crit Care. 2015;19:349.

6. Zhang W, Bai M, Yu Y, Li L, Zhao L, Sun S, Chen X. Safety and efficacy of regional citrate anticoagulation for continuous renal replacement therapy in liver failure patients: a systematic review and meta-analysis. Crit Care. 2019; 23(1):22.

7. Khadzhynov D, Dahlinger A, Schelter C, Peters H, Kindgen-Milles D, Budde K, Lehner L, Halleck F, Staeck O, Slowinski T. Hyperlactatemia, lactate kinetics and prediction of citrate accumulation in critically ill patients undergoing continuous renal replacement therapy with regional citrate anticoagulation. Crit Care Med. 2017;45(9):e941-6. 